\title{
CONTROLLING CHAOS IN A NEURAL NETWORK BASED ON THE PHASE SPACE CONSTRAINT
}

\author{
HE GUOGUANG \\ Department of physics, Zhejiang University, Zhe Da Road 38, \\ Hangzhou, 310027, China \\ CAO ZHITONG, CHEN HONGPING and ZHU PING \\ Institute of Applied physics, Zhejiang University, Zhe Da Road 38, \\ Hangzhou, 310027, China
}

Received 8 August 2002

\begin{abstract}
The chaotic neural network constructed with chaotic neurons exhibits very rich dynamic behaviors and has a nonperiodic associative memory. In the chaotic neural network, however, it is difficult to distinguish the stored patters from others, because the states of output of the network are in chaos. In order to apply the nonperiodic associative memory into information search and pattern identification, etc, it is necessary to control chaos in this chaotic neural network. In this paper, the phase space constraint method focused on the chaotic neural network is proposed. By analyzing the orbital of the network in phase space, we chose a part of states to be disturbed. In this way, the evolutional spaces of the strange attractors are constrained. The computer simulation proves that the chaos in the chaotic neural network can be controlled with above method and the network can converge in one of its stored patterns or their reverses which has the smallest Hamming distance with the initial state of the network. The work clarifies the application prospect of the associative dynamics of the chaotic neural network.
\end{abstract}

\section{Introduction}

A chaotic neural network constructed with chaotic neurons has exhibit very rich dynamic behaviors. Compared to the Hopfield neural network, the chaotic neural network possesses the characters of larger memory content and good tolerance, etc. Recently, it has received much attention because of its potential application of the associative dynamics in optimization and information processing, etc. ${ }^{1-3}$ The chaotic neural network has shown a nonperiodic associative memory, but its associative memory is realized in the chaos dynamic of the network. The outputs of the network are nonperiodic states which change continuously and can not be stabilized in one of its stored patterns. One therefore meets difficulties in the application of the associative memory in information processing. To achieve the information processing in the chaotic neural network, we should put the control on the network and let the network to be stable in an expected pattern. In our previous work, we have 
made an achievement in controlling the chaos of the network by using the pinning control. ${ }^{5}$ However, the controlled aim should be assigned prior in above method so that one still could not apply the associative memory of the chaotic neural network in information processing. In the work, we will propose a new control method focused on the chaotic neural network, in which the orbital in the phase space is constrained in a certain region. The computer simulation proves that the method works well. The chaotic neural network can be stabilized in a stored pattern or its reverse which has nearest Hamming distance with the initial state while no target need to be chose in advance.

In the second section we will give a simple description of the chaotic neural network used as a controlling object. Our new control method will be proposed in the following section. The computer simulation will be shown in the section too. The conclusion is in the fourth section.

\section{The Chaotic Neural Network Model and its Dynamics}

The chaotic neural network model used in the paper is constructed with 100 chaotic neurons. ${ }^{3,5}$ The dynamics of the ith chaotic neuron in the chaotic neural network is described as follows:

$$
\begin{aligned}
& x_{i}(t+1)=f\left[\eta_{i}(t+1)+\zeta_{i}(t+1)\right], \\
& \eta_{i}(t+1)=k_{f} \eta_{i}(t)+\sum_{j=1}^{100} w_{i j} x_{j}(t), \\
& \zeta_{i}(t+1)=k_{r} \zeta_{i}(t)+\alpha g\left[x_{i}(t)\right]+a_{i},
\end{aligned}
$$

where $\eta_{i}(t+1)$ and $\zeta_{i}(t+1)$ are the internal state variables for feedback input from the constituent neurons in network and refractoriness, respectively. $f(\cdot)$ and $g(\cdot)$ are the output function and the refractory function of the neuron, respectively. We take the output function of the neuron $f(x)$ as Sigmoid function with the steepness parameter $\epsilon$, i.e. $f(x)=1 /[1+\exp (-x / \epsilon)]$, refractoriness function as $g(x) \equiv x . \alpha$ is the refractory scaling parameter. $a_{i}(i=1,2,100)$ are the threshold of neurons. $k_{f}$ and $k_{r}$ are the decay parameters for the feedback inputs, and the refractoriness, respectively. $w_{i j}$ are synaptic weights to the ith constituent neuron from the jth constituent neuron, the weights are are defined according to the following symmetric auto-associative matrix of $n$ binary patterns:

$$
w_{i j}=\frac{1}{n} \sum_{p=1}^{n}\left(2 x_{i}^{p}-1\right)\left(2 x_{j}^{p}-1\right),
$$

where $x_{i}^{p}$ is the ith component of the pth binary pattern. In this way, the binary patterns can be stored as basal memory patterns. We use a picture composed of 10 by 10 matrix to show the stored patterns of the neural network constructed with 100 neurons. A neuron with its output $x_{i}$ equal to 1 , which means the neuron is 
"excited", is represented by a block "•" while a neuron with its output $x_{i}$ equal to 0 , which means the neuron is "restraining", is denoted by a dot ".". Four stored patterns employed in this paper are shown in Fig. 1.

When the decay parameters of the network are set to certain values, the network generates non-periodic sequential patterns including the stored ones as its output sequence. Figure 3 exhibits the sequences of the outputs for the network in which the parameters are taken as $\alpha=10.0, a_{i}=2.0, k_{f}=0.20$ and $k_{r}=0.95$ and the initial state is the one as shown in Fig. 2. One can finds that the outputs of the network exhibits the complex dynamic behave in time and space. The detail can be found in the Ref. 3. Though the stored patterns are included in the outputs, the network can not be stabilized in one stored pattern or near it. It is impossible to carry out the information processing in the network. In order to search the stored patterns involved in the network, one has to change the chaos dynamics, i.e. put the control on the chaotic neural network.

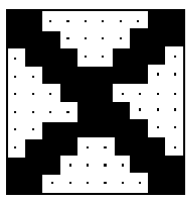

(a)

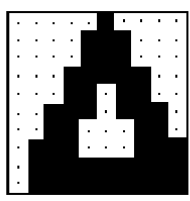

(b)

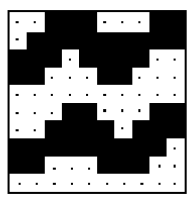

(c)

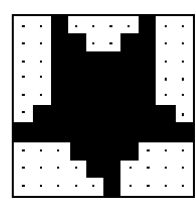

(d)

Fig. 1. Four stored patterns.

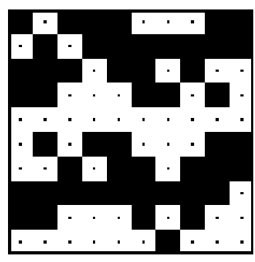

Fig. 2. Initial state.

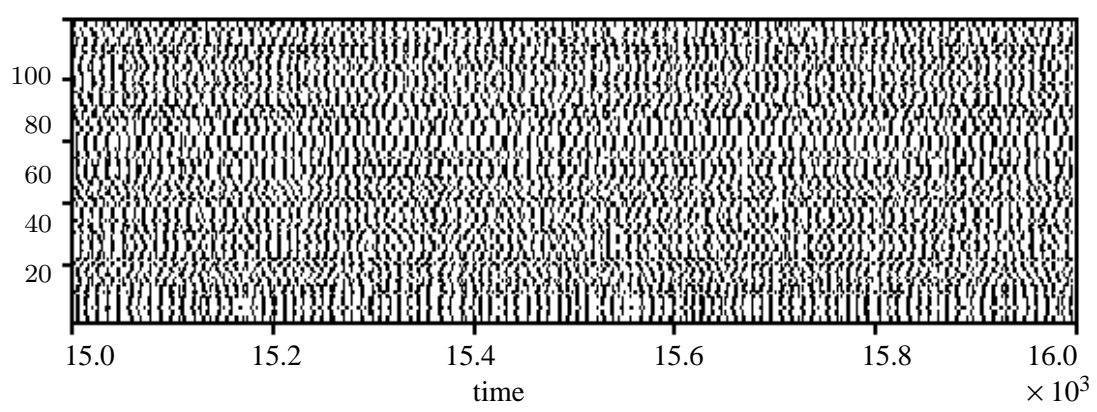

Fig. 3. The sequences of the output neurons. 


\section{Phase Space Constraint Control Method}

Since the pioneer work of Ott, Grebogi and Yorke (OGY), ${ }^{6}$ much attention has been paid on study of chaotic control. Several control methods were put forward, such as chaos synchronization, delayed self-controlling feedback, ${ }^{7}$ pinning control and phase space compression. ${ }^{8}$ One usually controls chaos of nonlinear systems for three purposes. The first one is to change a chaotic state into a stable or periodic state. The second is to let a stable or periodic state become chaotic. The final one is to transfer from one chaotic state to other. Our work is for the first purpose. Summing-up of all work in this field, there are two ways to make nonlinear systems converge in a stable or periodic state from a chaotic state: a feedback control and a nonfeedback control. In the former case, one chooses an unstable orbital of the chaotic systems as the goal of the controlling. The parts of outputs are taken as the feedback controlling variables. The controlling input is very small when system is well controlled. Our previous pinning control focused on the chaotic neural network is belong to this case. ${ }^{5}$ On the contrary, the latter is not related to a certain target. The controlling aim is achieved by limiting existent space of the chaos. In both case, the dynamical structure of the controlled system is different from the original one. The control method to be proposed here is belong to the latter. We change the dynamical structure of the chaotic neural network by constraining the existent area of its states. The chaotic motion can be controlled.

For the chaotic system described by Eqs. (1)-(3), the feedback input variable $\eta_{i}$ and the refractoriness variable $\zeta_{i}$ constitute its phase space. There are three ways to constrain the phase space of the system. Firstly one can only condense the feedback input variable $\eta_{i}$. Secondly only the refractoriness variable $\zeta_{i}$ is compressed. Finally both $\eta_{i}$ and $\zeta_{i}$ are controlled on certain area. Our simulation has proved that the chaotic system can quick converge in a stable state when the constraint is put on $\zeta_{i}$ only. Considering only the constraint of $\zeta_{i}$, the phase space constraint focused on the chaotic neural network is described with the following equation:

$$
\zeta_{i}(t+1)=\left\{\begin{array}{ll}
\zeta_{i}(t+1) & \zeta_{i \min }<\zeta_{i}(t+1)<\zeta_{\text {imax }} \\
\zeta_{\text {imax }} & \zeta_{i}(t+1) \geq \zeta_{\text {imax }} \\
\zeta_{\text {imin }} & \zeta_{i}(t+1) \leq \zeta_{\text {imin }}
\end{array} .\right.
$$

Equations (1)-(3) together with above equation constitute the dynamic model of the controlled chaotic neural network.

The controlled chaotic neural network can be stabilized in one of the stored patterns when suitable $\zeta_{\min }$ and $\zeta_{\max }$ are chosen. We show the sequences of the outputs for the controlled network with $\zeta_{\min }=-5.0$ and $\zeta_{\max }=5.0$ in Fig. 4 . One can find that the controlled network converges in a stable state after a few steps. The chaotic neural network changes from non-periodic chaotic dynamics to a stable orbital when its phase space is constrained. In our simulation, the network start from the state shown in Fig. 2 which is the stored pattern (c) with some errors. The stable state of the controlled network is the stored pattern (c). Further 
simulation has proved that the network can converge in the stored pattern (c) when $\zeta_{\max }$ is chosen less than 11.0 and $\zeta_{\min }$ larger than -7.4 . The values $\zeta_{\min }$ and $\zeta_{\max }$ are chosen in terms of the simulation experiments, which depended on the neural network model and the selected stored patterns.

When we chose some states which have a big Hamming distance with the four stored patterns as the initial state, the controlled network also quick converge in one of its stored pattern. The initial states and the corresponding stable outputs of the controlled network are given in Fig. 5. We list the Hamming distance between the initial states and the four stored patterns or their reverse patterns in Fig. 5 too. One can observe from Fig. 5 that starting from any initial state the controlled network can be stabilized in a stored pattern or its reverses which has the smallest Hamming distance with the initial state. Both the previous pinning control and the present constraint control are successful in controlling the chaos of the chaotic neural network. But the controlled aim in former one should be chose in advance, and the controlled network is employed lesser in practice. In the latter, no target needs to be set prior. The associative memory of the chaotic neural network can therefore be applied in the information processing by using the constraint control.

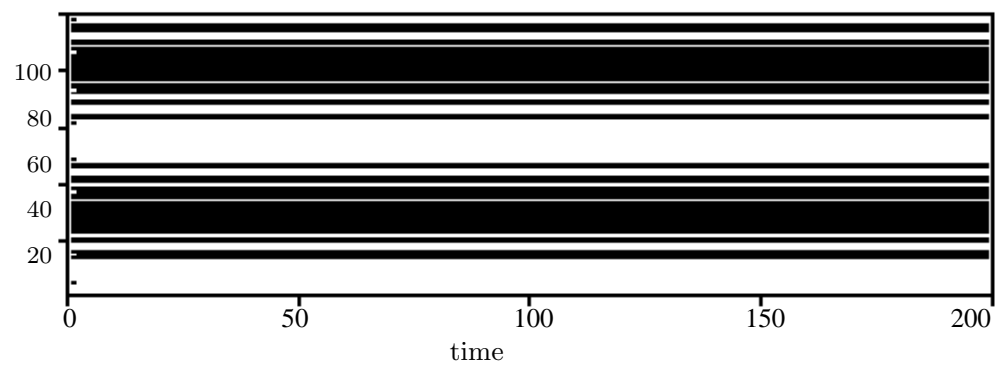

Fig. 4. The sequences of the outputs of neurons under control.

\begin{tabular}{|c|c|c|c|c|c|c|c|c|c|c|}
\hline \multicolumn{2}{|c|}{$\begin{array}{l}\text { Four stored and thei } \\
\text { reverses patterns }\end{array}$} & $\mathbf{X}$ & $\Delta$ & $\mathbf{\theta}$ & 2 & $\mathbf{E}$ & $\boldsymbol{H}$ & $\theta$ & 17 & $\begin{array}{l}\text { Output of } \\
\text { the netwrotk }\end{array}$ \\
\hline \multirow{4}{*}{$\begin{array}{l}\text { Iritial } \\
\text { state }\end{array}$} & & 46 & 62 & 42 & 59 & 54 & 38 & 58 & 41 & $\boldsymbol{H}$ \\
\hline & 国 & 41 & 53 & 41 & 52 & 59 & 47 & 59 & 48 & 3 \\
\hline & $\mathrm{E}$ & 44 & 42 & 50 & 33 & 56 & 58 & 50 & 67 & 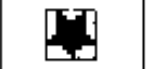 \\
\hline & $\therefore$ & 48 & 56 & 36 & 55 & 52 & 44 & 64 & 45 & 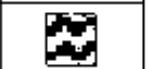 \\
\hline
\end{tabular}

Fig. 5. The output of the network under control in difference initial states. The numbers in the figure are the Hamming distance between the initial state and the stored or its reverse pattern. 


\section{Conclusion}

Though the chaotic neural network has large memory content and good tolerance, it is difficult to apply the network in optimization and information processing because of its chaotic motion. In order to exploit its potential application we must control the chaotic orbital of the network. It is well known that for nonlinear system the chaos is caused when its orbital is divergent or spread in one direction or ring. We have proposed a chaos control method of constraining phase space focused on the chaotic neural network. Actually, the divergence and spread of an orbital is limited in the method and the chaos is therefore controlled efficiently. The method is easier to be applied and its physical meaning is clearer compared to OGY method where one has to collect the characters about the Poincare map about an chaotic system. The controlled network can reach stability quickly in our method while it take much steps in some feedback control method. In addition, the control condition is wide in our method. When $\zeta_{\max }$ and $\zeta_{\min }$ are set in a broad area, the controlled network can be stabilized in one of its stored pattern. The computer simulation has proved that by employing our proposed constraint method the chaotic motion of the chaotic neural network can be controlled quickly and high efficiently and the network converge in one of its stored patterns or their reverse which has shortest Hamming distance with the initial state of the network. Our work point out a way to apply the associative dynamics of the chaotic neural network in information processing and optimization, etc.

\section{References}

1. CAO Zhitong, "The dynamic associative memory of chaotic neural", J. of Zhejiang University (Natural Science Edition), Supplement: p. 330-335 (in Chinese), 1998.

2. K. Aihara, T. Takabe and M. Toyoda, "chaotic neural networks". Phys Lett 144(6-7), 333-340 (1990).

3. M. Adachi and K. Aihara "Associative dynamics in a chaotic neural network", Neural Networks 10(1), 83-98 (1997).

4. E. R. Kobori, K. Ikoda and K. Nakayama, "A model of dynamic associative memory", IEEE International Conference on Neural Networks Conference Proceedings 2, 804-809 (1996).

5. He Guoguang and Cao Zhitong, "Controlling chaos in chaotic neural network", Acta Physica Sinica 50(11), p. 2103-2107 (in Chinese), 2001.

6. E. Ott, C. Grebogi and J. A. Yorke, "Controlling chaos", Phys. Rev. Lett. 64(11), p. 1196-1199 (1990).

7. Pyragas, K. "Continuous Control of Chaos by Self-Controlling Feedback", Phys. Lett. A170, p. 421-428 (1992).

8. X. S. Luo, "Using phase space compression to control chaos and hyperchaos", Acta Physica Sinica, 48(3), p. 402-406 (in Chinese), 1999. 